\title{
New distributional data on Geastrum (Geastraceae, Basidiomycota) from Brazil
}

\author{
Larissa Trierveiler-Pereira $^{1,4}$, Francisco de Diego Calonge ${ }^{2}$ and Iuri Goulart Baseia ${ }^{3}$
}

Recebido em 22/06/2010. Aceito em 9/06/2011

\begin{abstract}
RESUMO
(Novos dados de distribuição de Geastrum (Geastraceae, Basidiomycota) no Brasil). Os dados apresentados neste artigo são resultados de coletas feitas em campo e análise de espécimes fúngicos depositados no Herbário URM. As coletas foram realizadas em quatro remanescentes de mata atlântica no estado de Pernambuco, entre junho/2008 e maio/2009. A revisão de espécimes depositados no URM foi restrita a exsicatas brasileiras. Os espécimes de Geastrum foram examinados macro e microscopicamente segundo a metodologia tradicional do grupo. Geastrum javanicum e G. lloydianum são novos registros para a região Nordeste do Brasil. Oito espécies são novas ocorrências para os seguintes estados: Pará (G. fimbriatum), Ceará (G. lloydianum), Paraíba (G. fimbriatum, G. javanicum, G. schweinitzii e G. entomophilum), Pernambuco (G. lageniforme e G. triplex) e Rio de Janeiro (G. javanicum). Apresentamos neste artigo dez descrições completas de espécies de Geastrum, além de fotos das espécies e uma chave de identificação.
\end{abstract}

Palavras-chave: coleções de herbário, fungos “estrelas-da-terra”, fungos gasteróides, Gasteromycetes, taxonomia de fungos

\begin{abstract}
(New distributional data on Geastrum (Geastraceae, Basidiomycota) from Brazil). The data presented in this article are the results of field collections and the analysis of fungal specimens deposited in the URM Herbarium. The field trips were carried out in four Atlantic Forest remnants in the state of Pernambuco, between June 2008 and May 2009. The revision of specimens housed in the URM was restricted to the Brazilian exsiccatae. Geastrum specimens were examined macro- and microscopically following the traditional methodology used in the group. Geastrum javanicum and G. lloydianum are new records from the Northeastern Region of Brazil. Eight species are new records from the following states: Pará (G. fimbriatum), Ceará (G. lloydianum), Paraíba (G. fimbriatum, G. javanicum, G. schweinitzii and G. entomophilum), Pernambuco (G. lageniforme and G. triplex) and Rio de Janeiro (G. javanicum). In this article we present full descriptions for ten species of Geastrum with pictures and an identification key.
\end{abstract}

Key words: earthstar fungi, fungal taxonomy, gasteroid fungi, Gasteromycetes, herbarium collections

\section{Introduction}

Geastrum species, commonly known as 'earthstar' fungi, are easily recognized in the field by their exoperidium, which opens into a stellate shape. Some species, however, are found growing on rotten wood. The genus is characterized by an exoperidium divided into two or three layers, an endoperidium with or without a stalk, opening by an apical pore surrounded by an area called the peristome. The gleba is composed of abundant capillitium and basidiospores. (Ponce de León 1968, Sunhede 1989, Domínguez de To- ledo 1996, Kasuya et al. 2009). Currently, it is accepted that geastroid species are related to gomphoid/phalloid fungi (Hosaka et al. 2006).

According to Trierveiler-Pereira \& Baseia (2009a), forty Geastrum species are recorded from Brazil, but this number may be smaller since some synonyms were not considered in that article. Twenty-six Geastrum species are recorded from the state of Rio Grande do Sul, thanks especially to the great contribution of Rick's work (1961). Other recent Brazilian works concerning species of the genus are those by the following: Baseia \& Milanez (2003), Baseia et al. (2004),

\footnotetext{
1 Universidade Federal de Pernambuco, Programa de Pós-Graduação em Biologia de Fungos, Departamento de Micologia, Recife, PE, Brazil

2 Real Jardín Botánico, Consejo Superior de Ivestigaciones Cientificas, Madrid, Spain

3 Universidade Federal do Rio Grande do Norte, Departamento de Botânica, Ecologia e Zoologia, Natal, RN, Brazil

4 Author for correspondence: lt_pereira@yahoo.com.br
} 
Baseia \& Calonge (2006), Leite \& Baseia (2007) Leite et al. (2007a), Cortez et al. (2008a; 2008b), Fazolino et al. (2008), Trierveiler-Pereira et al. (2009), and Trierveiler-Pereira \& Baseia (2010).

The genus is frequent in the tropics and many herbarium collections registered under the name Geastrum have never been reviewed by specialists. Interesting data were published (Pereira et al. 2008, Gomes-Silva \& Gibertoni 2009) after revisions of fungal exsiccatae deposited in the URM Herbarium (Holmgren \& Holmgren 1998), including data on gasteromycete taxonomy (Drechsler-Santos et al. 2008, Trierveiler-Pereira \& Baseia 2009b). This herbarium, created in 1954, currently preserves more than 80.000 collections, the largest fungal collection in Latin America (Maia 2003).

This work aimed to contribute to the knowledge of Geastrum species distribution in Brazil, based on field collections and a review of herbarium material.

\section{Material and methods}

Field collections were carried out between June 2008 and May 2009 in four Atlantic Forest remnants in the state of Pernambuco, Brazil: Parque Ecológico Dois Irmãos (DI) $34^{\circ} 52^{\prime} 30^{\prime \prime} \mathrm{W}, 8^{\circ} 07^{\prime} 30^{\prime \prime}$, RPPN Carnijó (CA) - 350ㅗ'15”'W, $8^{\circ} 10^{\prime} 00^{\prime \prime}$, Parque Ecológico João de Vasconcelos Sobrinho

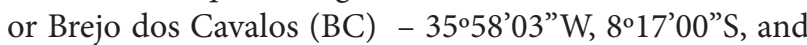

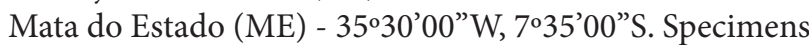
of gasteroid fungi were removed from the substratum with a knife, and then accommodated in plastic containers with individualized compartments (Fig. 2F).

The review of Geastrum material deposited in the URM Herbarium was restricted to Brazilian exsiccatae.

A taxonomic study was performed by observing macro- and microscopic features according to Miller \& Miller (1988) and Sunhede (1989). The basidiomata and endoperidium were measured including the size of the peristome. Colors were coded according to Kornerup \& Wanscher (1978) and referred to dry material, unless otherwise stated. Spore measurements included surface ornamentation.

Vouchers were deposited in the URM, but not all the examined material is listed in the results. Some collections were compared to specimens deposited in MA-Fungi (Holmgren \& Holmgren 1998).

\section{Results}

During this survey in the Atlantic Forest remnants in the state of Pernambuco, one hundred and forty-seven Geastrum specimens were collected, corresponding to nine species.

In the URM, forty-seven exsiccatae of Geastrum are registered, but three were not found (URM 941, 77072, 78646). Thirty-seven exsiccatae were collected in the state of Pernambuco. The forty-four revised materials corresponded to nine species of Geastrum.

\section{Key to the species of Geastrum}

1. Dark setae on the endoperidial surface present 9. Geastrum setiferum

1. Dark setae on the endoperidial surface absent

2. Peristome strongly plicate, apophysis present

6. G. lloydianum

2. Peristome fibrillose to faintly plicate, apophysis absent

3. Peristome delimited by a line and/or a groove ………………………………….........................................................

4. Basidiomata growing on rotten wood or dead leaves, with white subiculum .............................................................

5. Mycelial layer with long hairs, hirsute, basidiomata up to $2.5 \mathrm{~cm}$ diam.

5. Mycelial layer without hairs, basidiomata up to $1.5 \mathrm{~cm}$ diam

8. G. schweinitzii

4. Basidiomata growing on soil, without white subiculum

6. Pseudoparenchymatous layer breaking down at maturity, leaving a conspicuous collar around the endoperidium

10. G. triplex

6. Pseudoparenchymatous collar absent.

7. Basidiomata with arachnoid aspect, mycelial layer with longitudinal ridges, outer mycelial layer with thin-walled clamped hyphae

5. G. lageniforme

7. Basidiomata without such aspect, mycelial layer without longitudinal ridges, outer mycelial layer with simple-septate hyphae.....

7. G. saccatum

3. Peristome not delimited

8. External layer orange to brownish orange, with conical falsicles of hyphae and velutinous aspect, separating into two layers at maturity, growing on rotten wood and usually on a white subiculum 4. G. javanicum

8. External layer dirty white to yellowish, delicate, without conical hyphal pegs, growing on soil and without subiculum .............9

9. Exoperidium rays arched at maturity, endoperidium verruculose (presence of hyphal pegs). 1. G. entomophilum

9. Exoperidium with involute rays at maturity, endoperidium smooth (without hyphal pegs)

..2. G. fimbriatum 
1. Geastrum entomophilum Fazolino, Calonge \& Baseia, Mycotaxon 104: 450. 2008.

Fig. 1A

Expanded basidiomata $2.5-3.5 \mathrm{~cm}$ high $\times 2.8-3.3 \mathrm{~cm}$ diam. Exoperidium split into $4-6$ rays, first saccate, then becoming arched, non-hygroscopic; mycelial layer greyish yellow (KW 1B3), encrusted with debris, mycelial layer hyphae straight, solid or with narrow lumen, hyaline, 2.5-7 $\mu \mathrm{m}$ diam; fibrous layer greyish brown (KW 5D3), fibrous layer hyphae straight to sinuous, with narrow lumen, hyaline, 2-5 $\mu \mathrm{m}$ diam; pseudoparenchymatous layer pale yellow (KW $1 \mathrm{~A} 3$ ) to grayish orange (KW 5B3) when fresh, then golden brown (KW 5D7) to yellowish brown (KW 5F7) when dry, thick, persistent, pseudoparenchymatous hyphae round to irregular in shape, hyaline to yellowish, 27-68 × 16-36 $\mu \mathrm{m}$. Endoperidium brownish grey (KW $4 \mathrm{~F} 2)$ to dull yellow (KC 3B3), depressed-globose, $1.5-1.7 \mathrm{~cm}$ high $\times 1.5-1.7 \mathrm{~cm}$ diam., sessile, verruculose; endoperidial surface covered with protuberances made of projecting fascicles of hyphae; peristome concolor or slightly lighter than endoperidium, fibrillose to lacerate, not delimited. Capillitial hyphae straight, with narrow lumen and covered with amorphous material, golden brown, 4-10 $\mu \mathrm{m}$ diam. Basidiospores globose, very dark brown, verruculose, 3.5-4.5 $\mu \mathrm{m}$ diam. Growing on soil.

Material examined - BRAZIL. Pernambuco: Moreno, CA, 17/VI/2008, Trierveiler-Pereira \& Baltazar 24 (URM 80095); 08/VII/2008, Trierveiler-Pereira et al. 95 (URM 80096); Caruaru, BC, 20/VI/2008, Trierveiler-Pereira et al. 37 (URM 82053); Tamandaré, Reserva Biológica de Saltinho, 16/IV/2002, Baseia (URM 77075 - as G. pulverulentum); Igarassu, Mata da Usina São José, VII/2003, Baseia (URM URM 77233 - as G. pulverulentum); Paraíba: João Pessoa, Mata do Buraquinho UFPB, 12/VI/97, Baseia 210 (URM 75820 - as G. pulverulentum).

Taxonomic remarks - The species is characterized by arched rays at maturity, sessile endoperidium, verruculose endoperidial surface (due the presence of hyphal pegs) and a not delimited fibrillose peristome. The endoperidum is not always blackish as described in the original description (Fazolino et al. 2008), some specimens may show a pinkish white to dull yellow endoperidium (Trierveiler-Pereira \& Baseia 2010).

2. Geastrum fimbriatum Fr., Syst. mycol. (Lundae) 3(1): 16. 1829.

Fig. 1B

Expanded basidiomata $1.5-3.4 \mathrm{~cm}$ high $\times 1.2-3.1 \mathrm{~cm}$ diam. Exoperidium split into 5-7 rays, first saccate, then becoming involute, rarely arched, non-hygroscopic; mycelial layer yellowish grey (KC 2B2) when fresh, then blond (KC 4C4) when dry, strongly encrusted with debris and usually peeling off at maturity, mycelial layer hyphae tortuous, with narrow lumen, golden yellow, 3-8 $\mu \mathrm{m}$ diam; fibrous layer pale yellow (KC 2A3), fibrous layer hyphae more less straight, rarely branched, with narrow lumen, hyaline to yellow, 2-5 $\mu \mathrm{m}$ diam.; pseudoparenchymatous layer pale grey (KC 1B1) to orange white (KC 5A2) when fresh, then greyish yellow (KC 4C4) when dry, pseudoparenchymatous hyphae round to isodiametric, yellowish, $30-56 \times 23-42 \mu \mathrm{m}$. Endoperidium olive brown (KW 4E4), globose, subglobose to depressed-globose, $0.5-1.5 \mathrm{~cm}$ high $\times 0.7-1.9 \mathrm{~cm}$ diam., sessile; peristome concolor with endoperidium, fibrillose, not delimited, shortly conical or truncate at the apex, up to $0.1 \mathrm{~cm}$ high. Capillitial hyphae sinuous, with narrow lumen, often covered by amorphous material, golden yellow to olive yellow, 1.5-4 $\mu \mathrm{m}$ diam. Basidiospores globose, yellowish brown, with more or less columnar ornamentation, 4-5 $\mu \mathrm{m}$ diam. Growing on soil.

Material examined - BRAZIL. Pernambuco: São Vicente Férrer, ME, 26/VI/2008, Trierveiler-Pereira et al. 41-44, 46 (URM 82037-82040, 82041); 19/VII/2008, TrierveilerPereira et al. 112, 118-119, 122-123 (URM 82043, 8204482045, 82046-82047), 28/VIII/2008, Trierveiler-Pereira \& Baltazar 166 (URM 82048); Caruaru, BC, 12/VII/2008, Trierveiler-Pereira et al. 107 (URM 82042); Recife, DI, 03/IX/1951, Silva (URM 466 - as G. pectinatus); Jardim Botânico de Curado, 11.VII.1995, Kimbrough (URM 75766 -as G. velutinus); Paraíba: João Pessoa, Mata do Buraquinho UFPB, 31/VI/1997, Baseia 127 (URM 75816); Pará: Belém, 04/I/1961, Batista (URM 47769 - as G. triplex).

Taxonomic remarks - Geastrum fimbriatum is distinguished from other species in the genus by its involute rays at maturity; mycelial layer strongly encrusted with debris; pinkish exoperidium when fresh; sessile and blackish endoperidium; fibrillose and not delimited peristome. This species shares a number of features with G. javanicum (see discussion below), but the latter is found growing on rotten wood. A small pseudoparenchymatous collar around the endoperidial body may be observed in some specimens (Sunhede 1989), but it is not so prominent as that found in G. triplex. According to Calonge (1998), G. badium is morphologically similar to G. fimbriatum but the former has a plicate peristome.

3. Geastrum hirsutum Baseia \& Calonge, Mycotaxon 95: 302. 2006.

Fig. 1C

Immature basidiomata subglobose to obovoid, 0.7-1.4 $\mathrm{cm}$ high $\times 0.5-1.5 \mathrm{~cm}$ diam., external layer hirsute, dark yellow (KC 5D4) to yellowish brown (KC 5E4). Expanded basidiomata $0.9-1.5 \mathrm{~cm}$ high $\times 1.1-2.5 \mathrm{~cm}$ diam. Exoperidium split into 5-8 rays, saccate, non-hygroscopic; mycelial layer hirsute, not encrusted with debris, usually peeling off at maturity, yellowish brown (KC 5E4), mycelial layer hyphae more or less straight, with narrow lumen, yellowish to brownish, 4-8 $\mu \mathrm{m}$ diam.; fibrous layer greyish (KC 4C5), fibrous layer hyphae straight to sinuous, solid or with very narrow lumen, hyaline, 3-7.5 $\mu \mathrm{m}$ diam; pseudoparenchymatous layer yellowish brown (KC 1B1), pseudoparenchyma- 
tous hyphae round to isodiametric, hyaline, $21-41 \times 20-35$ $\mu \mathrm{m}$. Endoperidium grey (KW 5D1), subglobose, 0.5-1.0 $\mathrm{cm}$ high $\times 0.8-1.1 \mathrm{~cm}$ diam., sessile; peristome brownish beige (KC 6E3), fibrillose, delimited by a brownish grey (KC 6C2) line. Capillitial hyphae sinuous, with narrow lumen, yellowish to brownish, 3-6 $\mu \mathrm{m}$ diam. Basidiospores globose, golden brown, with columnar ornamentation, 3.5-4 $\mu \mathrm{m}$ diam. Growing on a white subiculum on rotten wood.

Material examined - BRAZIL. Pernambuco: Recife, DI, 13/VI/2008, Trierveiler-Pereira et al. 08 (URM 80278); 07/VII/2008, Trierveiler-Pereira et al. 59-60, 69 (URM 80280-80281, 80282); 12/VIII/2008, Trierveiler-Pereira et al. 131 (URM 80285); 21/VI/2003, Baseia (URM 78711 holotype); 21/VI/2003, Baseia (MA-Fungi 67886 - paratype); Moreno, CA, 17/VI/2008, Trierveiler-Pereira \& Baltazar 13 (URM 80279); 08/VII/2008, Trierveiler-Pereira et al. 79, 97 (URM 80283, 80284); 14/VIII/2008, Trierveiler-Pereira et al. 145, 154, 160 (URM 80286, 80287, 80288); 23/IX/2008, Trierveiler-Pereira \& Baltazar 185 (URM 80289); 16/X/2008, Trierveiler-Pereira \& Baltazar 202 (URM 80290); 21/V/2009, Baltazar et al. 224 (URM 80291); São Vicente Férrer, ME, 28/V/2009, Trierveiler-Pereira et al. 238 (URM 80292).

Taxonomic remarks - This species was originally described based on Brazilian specimens and its most important characteristic is the presence of golden yellow hairs on the external layer (Baseia \& Calonge 2006). Specimens of $G$. hirsutum grow in habitats similar to those of G. schweinitzii, but the latter usually produces smaller basidiomata. Geastrum hirsutum was originally described growing on termite nests, but all specimens collected for this work were found growing on rotten wood.

4. Geastrum javanicum Lév., Champ. Mus. Paris: 161. 1846. Fig. 1D

Immature basidiomata globose to subglobose, with or without a pointed apex, $1.3-2.1 \mathrm{~cm}$ high $\times 1.1-2.0 \mathrm{~cm}$ diam., external layer light orange (KC 5A5) to yellowish brown (KC 5D5), velutinous due to the presence of conical fascicles of hyphae up to $0.7 \mathrm{~cm}$ high $\times 0.5 \mathrm{~cm}$ diam. Expanded basidiomata $1.7-2.9 \mathrm{~cm}$ high $\times 1.3-5.1 \mathrm{~cm}$ diam., sometimes with a short stipe up to $3.0 \mathrm{~cm}$ high. Exoperidium split into 5-7 rays, saccate, non-hygroscopic; mycelial layer not persistent; fibrous layer orange white (KC 5A2), macroscopically similar to the external layer of immature basidiomata, fibrous layer hyphae more or less straight, solid or with very narrow lumen, hyaline to slightly yellowish, 3-4 $\mu \mathrm{m}$ diam.; pseudoparenchymatous layer greyish orange ( $\mathrm{KC}$ $5 \mathrm{~B} 4$ ) to brownish orange (KC 5C4) when fresh, then greyish brown (KC 5D3) when dry, pseudoparenchymatous hyphae round, yellowish, 20-45×23-35 $\mu \mathrm{m}$. Endoperidium greyish brown (KW 5D3) to yellowish brown (KW 5E4), globose, subglobose to depressed-globose, $0.7-1.7 \mathrm{~cm}$ high $\times 1.2-1.8$ $\mathrm{cm}$ diam., sessile; peristome concolor or slightly darker than endoperidium, fibrillose, not delimited, up to $1.5 \mathrm{~mm}$ high. Capillitial hyphae straight to more or less sinuous, solid or with very narrow lumen, yellowish brown to olive brown, 2-7 $\mu \mathrm{m}$ diam. Basidiospores globose, brownish, with more or less columnar ornamentation, $4-5 \mu \mathrm{m}$ diam. Growing on a white subiculum on rotten wood.

Material examined - BRAZIL. Pernambuco: Recife, DI, 12/VIII/2008, Trierveiler-Pereira et al. 130 (URM 82033); 15/VII/1948, Lima (URM 376 - as G. triplex); 03/ IX/1951, Silva (URM 465 - as G. arenarius); 08/VIII/1954, Soares (URM 840 - as G. saccatum); I/2004, Baseia (URM 77569 - as G. sp); Campus UFPE, 17/VI/1995, Kimbrough (URM 75719 - as G. mirabilis); Moreno, CA, 17/VI/2008, Trierveiler-Pereira \& Baltazar 19, 23 (URM 82025, 82026); 08/VII/2008, Trierveiler-Pereira et al. 72, 85, 91 (URM 82028, 82029, 82030); 14/VIII/2008, Trierveiler-Pereira et al. 146 (URM 82034); Caruaru, BC, 20/VI/2008, TrierveilerPereira et al. 33 (URM 82027); 12/VII/2008, TrierveilerPereira et al. 106 (URM 82031); São Vicente Férrer, ME, 19/VII/2008, Trierveiler-Pereira et al. 114 (URM 82032); 28/V/2009, Trierveiler-Pereira et al. 239 (URM 82036); Vitória, 26/IX/1954, da Silva (URM 1013 - as G. drummondii); Paraíba: João Pessoa, Mata do Buraquinho UFPB, 28/IV/1997, Baseia 124 (URM 75817 - as G. cf. arenarius); Rio de Janeiro: Rio de Janeiro, Botafogo, 1914, Torrend (URM 8579 - as G. velutinum); Minas Gerais: Santa Maria, IV/1914, Torrend 4.1914 ( URM 9551 - as G. velutinum).

Taxonomic remarks - This species is characterized by an external layer with conical hyphal fascicles and velutinous aspect, darkish and sessile endoperidium, fibrillose and not delimited peristome. The fibrous layer divides into two layers at maturity. Geastrum javanicum shares some features with G. fimbriatum but they can be separated by habitat and external layer characteristics.

5. Geastrum lageniforme Vittad., Monogr. Lycoperd.: 1617. 1842.

Fig. 1E

Expanded basidiomata $0.7-1.3 \mathrm{~cm}$ high $\times 2.4-4.5 \mathrm{~cm}$ diam, with arachnoid aspect. Exoperidium split into 6-8 rays, saccate, rays are long, slender and with longitudinal ridges on the external layer, non-hygroscopic; mycelial layer encrusted with few debris, partially persistent at maturity, yellowish brown (KC 5E6) to brown (KC 6E6); mycelial layer hyphae tortuous, solid or with narrow lumen, slightly yellowish, 2-4 $\mu \mathrm{m}$ diam., with or without clamp connection; fibrous layer yellowish white (KC 1A2), fibrous layer hyphae straight to tortuous, solid or with narrow lumen, hyaline to slightly yellowish, 2-9 $\mu \mathrm{m}$ diam.; pseudoparenchymatous layer yellowish grey (KC 3B2) when fresh, then dark brown (KC 5D4) when dry, pseudoparenchymatous hyphae round, yellowish, 32-104 $\times 27-56 \mu \mathrm{m}$. Endoperidium pale orange (KW 5A3) when fresh, then blond (KW 4C4), globose, 0.8$1.2 \mathrm{~cm}$ high $\times 0.9-1.3 \mathrm{~cm}$ diam., sessile; peristome concolor with endoperidium, fibrillose, up to $0.2 \mathrm{~cm}$ high, delimited by a whitish line. Capillitial hyphae straight to more or less sinuous, with narrow lumen, covered with amorphous 
material, yellowish, 2.5-7 $\mu \mathrm{m}$ diam. Basidiospores globose, yellowish brown, with columnar ornamentation, $4-5 \mu \mathrm{m}$ diam. Growing on soil.

Material examined - BRAZIL. Pernambuco: São Vicente Férrer, ME, 26/VI/2008, Trierveiler-Pereira et al. 54 (URM 82018); 28/V/2009, Trierveiler-Pereira et al. 237 (URM 82019).

Additional material examined - ESPANHA. Burgos: Quintana del Pidio, 22/XI/1991, Parra (MA-Fungi 30752).

Taxonomic remarks - This species is very similar to $G$. saccatum. Both species have saccate basidiomata, sessile endoperidium, fibrillose and delimited peristome. Geastrum lageniforme usually has longer and more slender rays and longitudinal ridges in the external layer. However, $G$. saccatum may also have such ridges. According to Sunhede (1989), the two species can be distinguished by the presence of clamped hyphae in the external mycelial layer, which only occur in G. lageniforme. These hyphae were observed in the examined material, but they are not always easy to find. Ponce de León (1968) considered G. lageniforme a synonym of G. indicum (Klotzsch) Rauschert.

\section{Geastrum lloydianum Rick, Brotéria 5: 26. 1906.} Fig. $1 \mathrm{~F}$

Expanded basidiomata $2.7-4.4 \mathrm{~cm}$ high $\times 2.0-4.7$ $\mathrm{cm}$ diam. Exoperidium split into 5-9 rays, arched, nonhygroscopic; mycelial layer encrusted with debris, usually peeling off at maturity, yellowish white (KC 2A2); mycelial layer hyphae narrow, sinuous, slightly thick-walled, yellowish, 1-2 $\mu \mathrm{m}$ diam.; fibrous layer orange (KC 5A2), fibrous layer hyphae more or less straight to very tortuous, with narrow lumen, hyaline to slightly yellowish, $2-7 \mu \mathrm{m}$ diam.; pseudoparenchymatous layer yellowish white (KC 1A2) to yellowish brown $(\mathrm{KC}$ DD5, 6E6) when fresh, then yellowish brown (KC 5E5) when dry, pseudoparenchymatous hyphae round, yellowish, 22-50 × 19-42 $\mu \mathrm{m}$. Endoperidium yellowish brown (KW 5E4), covered or not with crystalline matter when recently exposed, globose to subglobose, $1.1-1.5 \mathrm{~cm}$ high $\times 1.3-1.9 \mathrm{~cm}$ diam., sessile or with a very short stipe, $0.2 \mathrm{~cm}$ high $\times 0.2-0.3 \mathrm{~cm}$ diam.; apophysis may be observed after the material is dried; peristome yellowish brown (KC 5F8), strongly plicate, conical, $0.25-0.6 \mathrm{~cm}$ high $\times 0.3-0.5 \mathrm{~cm}$ diam., delimited by a black line and/or a groove. Capillitial hyphae straight, solid or with very narrow lumen, usually covered with amorphous material, golden to yellowish brown, 3-10.5 $\mu \mathrm{m}$ diam. Basidiospores globose, brownish, with more or less columnar ornamentation, 4-4.5 $\mu \mathrm{m}$ diam. Growing on soil.

Material examined - BRAZIL. Pernambuco: Recife, DI, 07/VII/2008, Trierveiler-Pereira et al. 57 (URM 82011); 16/IX/2008, Trierveiler-Pereira et al. 173 (URM 82016); 10/VII/1949, Batista (URM 595 - as G. coronatum); VII/2002, Baseia (URM 77225 - as G. harriotii); Moreno, CA, 08/VII/2008, Trierveiler-Pereira et al. 100 (URM 82012); São Vicente Férrer, ME, 19/VII/2008, Tri-
erveiler-Pereira \& et al. 129 (URM 82013); 28/VIII/2008, Trierveiler-Pereira \& Baltazar 165, 167 (URM 82014, 82015); 18/IX/2008, Trierveiler-Pereira \& Baltazar 180 (URM 82017); Ceará: Fortaleza, 29/II/1962, Batista (URM 47778 - as G. harriotii).

Additional material examined - BRAZIL. Pernambuco: Cabo de Santo Agostinho, Reserva Ecológica de Gurjaú, 24/ VI/1998, Baseia (UFRN-Fungos 225); VENEZUELA. Sucre: Sabacual, 11/III/1989, Verde de Millán (MA-Fungi 34139). COSTA RICA, López 6029 (MA-Fungi 68706).

Taxonomic remarks - Geastrum lloydianum (= G. harriotti Lloyd) was described based on Brazilian specimens and it is known from tropical America, Australia and Sri Lanka (Ponce de León 1968). Previous to the present study, the species was only known from Southern Brazil in the state of Rio Grande do Sul (Rick 1961).

Geastrum badium Pers. (= G. elegans Vittad.) is a very similar species, but it has involute rays, sessile endoperidium, and lacks apophysis. Ponce de León (1968) reported that G. badium has hygroscopic basidiome and endoperidial body with granular surface. Leite et al. (2007a) recorded G. elegans from Pernambuco; however, a careful analysis of the material revealed that it actually corresponds to $G$. lloydianum.

G. setiferum resembles G. lloydianum in basidiomata size and the arched exoperidium; however, the endoperidium is fibrillose to faintly plicate and dark brown setae are present in the endoperidial surface.

7. Geastrum saccatum Fr., Syst. mycol. (Lundae) 3(1): 16. 1829.

Fig. 2A

Expanded basidiomata $0.8-2.1 \mathrm{~cm}$ high $\times 3.9-5.0 \mathrm{~cm}$ diam. Exoperidium split into 6-8 rays, saccate, some rays are split at the apex, involute or not, non-hygroscopic; mycelial layer without encrusted debris, greyish yellow (KC 4C4); mycelial layer hyphae straight, solid or with narrow lumen, yellowish, 2-10 $\mu \mathrm{m}$ diam.; fibrous layer greyish orange (KC 5B4), fibrous layer hyphae straight to sinuous, solid or with narrow lumen, yellowish, $2-3 \mu \mathrm{m}$ diam.; pseudoparenchymatous layer yellowish brown (KC 5E5), pseudoparenchymatous hyphae round, yellowish, $17-44 \times 10-29 \mu \mathrm{m}$. Endoperidium greyish green (KC 1C3), depressed-globose, $0.5-1.5 \mathrm{~cm}$ high $\times 0.7-1.6 \mathrm{~cm}$ diam., sessile; peristome slightly darker than endoperidium, greyish yellow (KC 2C3), fibrillose, up to $0.05 \mathrm{~cm}$ high, delimited by a dull yellow (KC 3B3) line. Capillitial hyphae straight, with wide or narrow lumen, covered with amorphous material, yellowish to slightly brownish, 2-6 $\mu \mathrm{m}$ diam. Basidiospores globose, brownish, ornamented with dense high columns, 4-5.5 $\mu \mathrm{m}$ diam. Growing on soil.

Material examined - BRAZIL. Pernambuco: São Vicente Férrer, ME, 26/VI/2008, Trierveiler-Pereira et al. 40 (URM 82020); 19/VII/2008, Trierveiler-Pereira et al. 127 (URM 82022); 28/VIII/2008, Trierveiler-Pereira \& Baltazar 

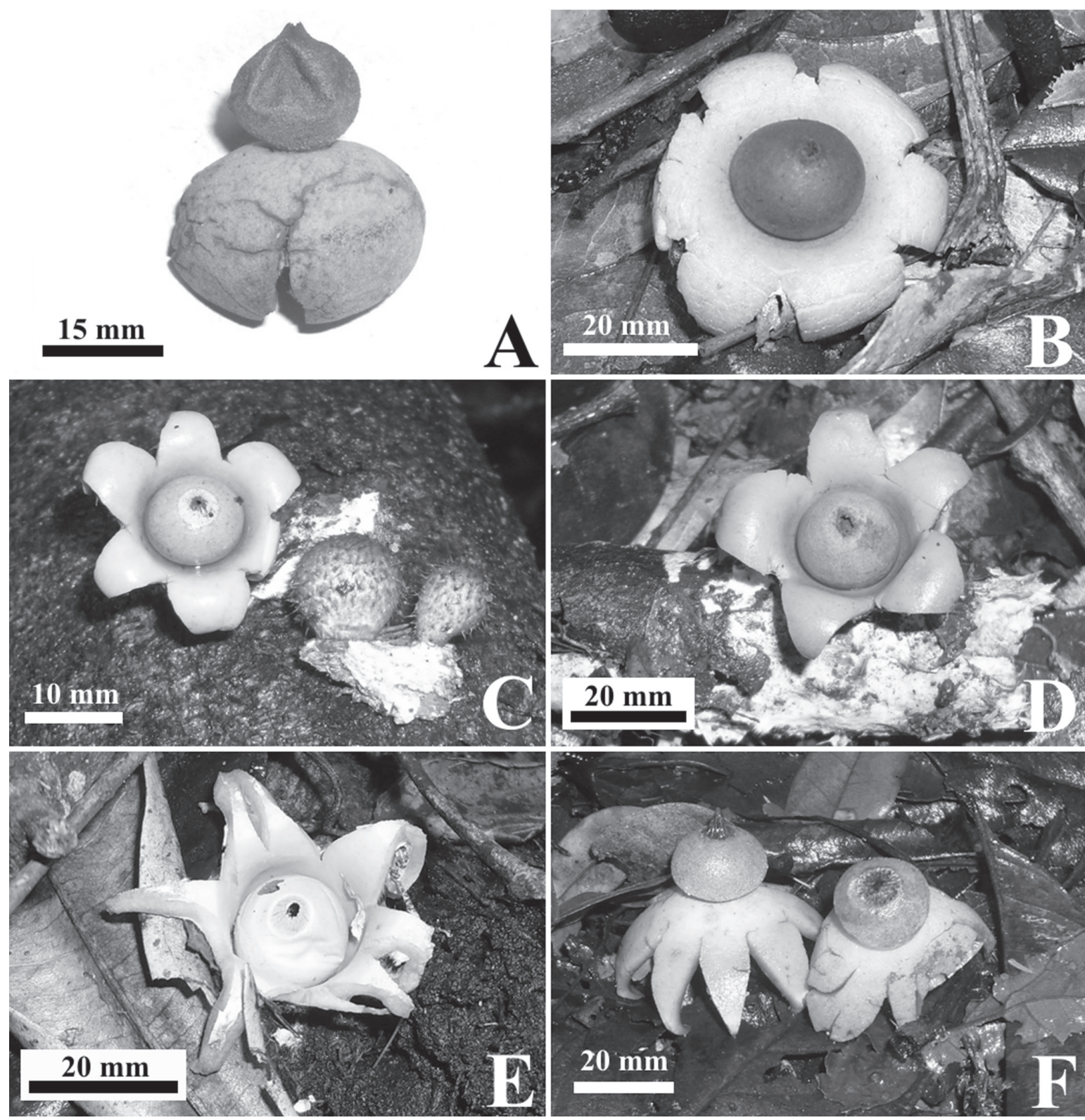

Figure 1. Basidiomata of Geastrum species. A. G. entomophilum. B. G. fimbriatum. C. G. hirsutum. D. G. javanicum. E. G. lageniforme. F. G. lloydianum.

171 (URM 82023); Caruaru, BC, 12/VII'12008, TrierveilerPereira et al. 104 (URM 82021); Recife, DI, 31/VIII/1951, Silva (URM 655 - as G. radicans); Campus da UFPE, 22/ VI/1995, Kimbrough (URM 75718).

Taxonomic remarks - Geastrum saccatum was described based on Brazilian specimens. However, the holotype of the species is missing (Sunhede 1989). According to Baseia et al. (2003), G. saccatum can be distinguished by its saccate exoperidium, sessile endoperidium, fibrillose and delimited peristome. This species is very similar to G. lageniforme (see discussion above).

8. Geastrum schweinitzii (Berk. \& M.A. Curtis) Zeller, Mycologia 40(6): 649. 1948.

Fig. 2B
Immature basidiomata globose to subglobose, $0.9-0.9$ $\mathrm{cm}$ high $\times 0.4-1.0 \mathrm{~cm}$ diam., external layer smooth or minute hirsute, yellowish grey (KC 2B2) to greyish (KC 2B1). Expanded basidiomata $0.5-1.2 \mathrm{~cm}$ high $\times 0.6-1.5 \mathrm{~cm}$ diam, rarely with very short stipe. Exoperidium split into 5-7 short rays, saccate, non-hygroscopic; mycelial layer without encrusted debris, yellowish grey (KC 4C4); mycelial layer hyphae straight, solid or with narrow lumen, hyaline, 2.5-11 $\mu \mathrm{m}$ diam.; fibrous layer greyish yellow (KC 1B5), fibrous layer hyphae straight, solid or with narrow lumen, hyaline to yellowish, $2-5 \mu \mathrm{m}$ diam.; pseudoparenchymatous layer reddish brown (KC 8E4, 9D4), pseudoparenchymatous hyphae round to isodiametric, hyaline to yellowish, 35-66 $\times 21-50 \mu \mathrm{m}$. Endoperidium grey (KC 7C1), greyish brown (KC 7F2) to brown (KC 6E4), globose to depressed-globose, 
0.5-0.7 $\mathrm{cm}$ high $\times 0.5-0.7 \mathrm{~cm}$ diam., sessile; peristome grey (KC 7B1, 6C1, 5B1), reddish white (KC 7A2), orange white (KC 6A2) to brownish beige (6E3), fibrillose, delimited by a greyish brown (KC 8D3) line. Capillitial hyphae straight, solid or with narrow lumen, brownish, 2-5 $\mu \mathrm{m}$ diam. Basidiospores globose, yellowish to golden brown, verrucose, 2-5 $\mu \mathrm{m}$ diam. Growing on a white subiculum on rotten wood and dead leaves.

Material examined - BRAZIL. Pernambuco: Recife, DI, 13/VI/2008, Trierveiler-Pereira et al. 01-06 (URM 8012480129); 07/VII/2008, Trierveiler-Pereira et al. 62-66, 70 (URM 80137-80141); 12/VIII/2008, Trierveiler-Pereira et al. 132-136, 138-141, 143 (URM 80160-80164, 80165-80168, 80169); 31/VIII/1951, Batista (URM 656 - as G. saccatum); 12/ VI/1957, Batista (URM 47641 - as G. minutus); 24/VII/1955, Batista (URM 47784 - as G. coronatus); VII.2002, Baseia (URM 77230); Moreno, CA, 17/VI/2008, Trierveiler-Pereira \& Baltazar 12, 14, 16, 18, 21-22, 26 (URM 80130, 80131, 80132, 80133, 80134-80135, 80136); 08/VII/2008, TrierveilerPereira et al.70-77, 81, 86-90, 92, 94, 98-99 (URM 8014280147, 80148, 80149-80153, 80154, 80155, 80156-80157); São Vicente Férrer, ME, 19/VII/2008, Trierveiler-Pereira et al. 113, 117 (URM 80158, 80159); 18/IX/2008, TrierveilerPereira \& Baltazar 179, 182 (URM 80183, 80184); 28/V/2009, Trierveiler-Pereira et al. 234, 236 (URM 80208, 80209); Cabo de Santo Agostinho: Reserva de Gurjaú, 29/VI/2002, Baseia (URM 77076); Caruaru, 14/I/1959, Batista (URM 47819 - as G. saccatum); Santo Amaro, Reserva Florestal da Usina Serra Grande, VI/2003, Baseia (URM 77548); Reserva Biológica de Pedra Talhada, IV/2003, Baseia (URM 77552); Igarassu, Refugio Ecológico Charles Darwin, IV/2003, Baseia (URM 77556); Tamandaré, Saltinho, V/2003, Baseia (URM 77557); Paraíba: João Pessoa, Mata do Buraquinho UFPB, 28/IV/1997, Baseia 127 (URM 75818); Bahia: Salvador, Rio Vermelho, IV/1915, Torrend (URM 9022 - as G. mirabilis).

Taxonomic remarks - Geastrum schweinitzii is a very minute species that is relatively easy to find in the field due to its caespitose to gregarious habit and abundant subiculum covering the substrata (Baseia et al. 2003). Geastrum pleosporum Douanla-Meli, described from Cameroon (Douanla-Meli et al. 2005), is macroscopically similar to $G$. schweinitzii, but the former has subsmooth basidiospores with varying shape: oblong, ovoid, cylindrical, elliptic to clubshaped. Geastrum schweinitzii resembles Geastrum hirsutum (see discussion above).

9. Geastrum setiferum Baseia, Mycotaxon 84: 136. 2002. Fig. 2C, 2D

Expanded basidiomata $2.4 \mathrm{~cm}$ high $\times 5.9 \mathrm{~cm}$ diam. Exoperidium split into 6 rays, applanate to slightly arched, nonhygroscopic; mycelial layer pale yellow (KW 4A3), peeling off at maturity, mycelial layer hyphae sinuous, thick-walled or solid, hyaline to yellowish, 1.5-3 $\mu \mathrm{m}$ diam; fibrous layer greyish yellow (KW 4C5), fibrous layer hyphae straight to sinuous, with very narrow lumen or solid, yellowish, 4-13 $\mu \mathrm{m}$ diam; pseudoparenchymatous layer yellowish brown (KW 5D5), persistent, pseudoparenchymatous hyphae isodiametric, yellowish brown to brown, 33-93 $\times 25-48 \mu \mathrm{m}$. Endoperidium light brown (KW 5D5), depressed-globose, $1.6 \mathrm{~cm}$ high $\times 2.2 \mathrm{~cm}$ diam., with a short stipe $1.5 \mathrm{~mm}$ high, apophysis present; endoperidial surface covered with yellowish brown to brown setae, with very narrow lumen, 162-215 $\times 34-47$; peristome concolor with endoperidium, fibrillose to slightly plicate, weakly delimited. Capillitial hyphae straight to sinuous, with narrow lumen or solid, covered with amorphous material, golden brown, $2.5-8 \mu \mathrm{m}$ diam. Basidiospores globose, brownish, ornamented with more or less columnar ornamentation, 3.5-4.0 $\mu \mathrm{m}$ diam. Growing on soil.

Material examined - BRAZIL. Pernambuco: Serra Negra, 28/V/2002, Baseia (URM 77077 - paratype).

Taxonomic remarks - The most striking characteristic of this species is the presence of dark brown setae in the endoperidial surface. The exoperidium appear saccate in drawings and photos (Baseia \& Milanez 2002, Baseia et al. 2006, Leite et al. 2007b) but this is probably the initial state of the peridial aperture. Herbarium materials have exoperidium rays applanate to arched. The endoperidium is not sessile as described originally; instead, it has a short stipe (Fig. 2D). It is also possible to observe the presence of apophysis on dried material. The peristome is a transition between fibrillose and plicate and is weakly delimited.

10. Geastrum triplex, Jungh., Tijdschr. Nat. Gesch. Physiol. 7: 287. 1840.

Fig. 2E

Immature basidiomata onion-shaped, with longitudinal ridges from the apex to the base, $3.0 \mathrm{~cm}$ high $\times 3.4 \mathrm{~cm}$ diam., yellowish brown (KC 5E5), without encrusted debris. Expanded basidiomata 4.7-6.4 cm diam. Exoperidium split into 5-8 rays, saccate, rays involute, non-hygroscopic. Mycelial layer with few encrusted debris, usually peeling off at maturity, golden (KC 4C6), greyish yellow (KC4C7) to olive (KC 3D3), mycelial layer hyphae straight to sinuous, with narrow lumen, yellowish, 3-7 $\mu \mathrm{m}$ diam.; fibrous layer greyish yellow (KC 1B4) to golden (KC 4C6), fibrous layer hyphae straight to sinuous, with narrow lumen, rarely solid, hyaline to slightly yellowish, 3-6 $\mu \mathrm{m}$ diam.; pseudoparenchymatous layer light brown (KC 7D4), brown (KC 7E5) to dark brown (KC 7F5) when fresh, forming a prominent collar around the endoperidium, pseudoparenchymatous hyphae round to isodiametric, 13-37 ×9-32 $\mu \mathrm{m}$. Endoperidium yellowish brown (KW 5F5) to dark brown (KW 6F4, 6F5) when fresh, globose, $1.8-2.4 \mathrm{~cm}$ high $\times 1.9-2.5 \mathrm{~cm}$ diam., sessile; peristome dark brown (KC 6F4), fibrillose, delimited by a light brown line. Capillitial hyphae straight, solid or with narrow lumen, yellowish to brownish, 2-6 $\mu \mathrm{m}$ diam., covered or not with amorphous material. Basidiospores golden brown to brownish, densely ornamented with more or less columnar processes, 4.5-5.5 (-6) $\mu \mathrm{m}$ diam. Growing on soil. 

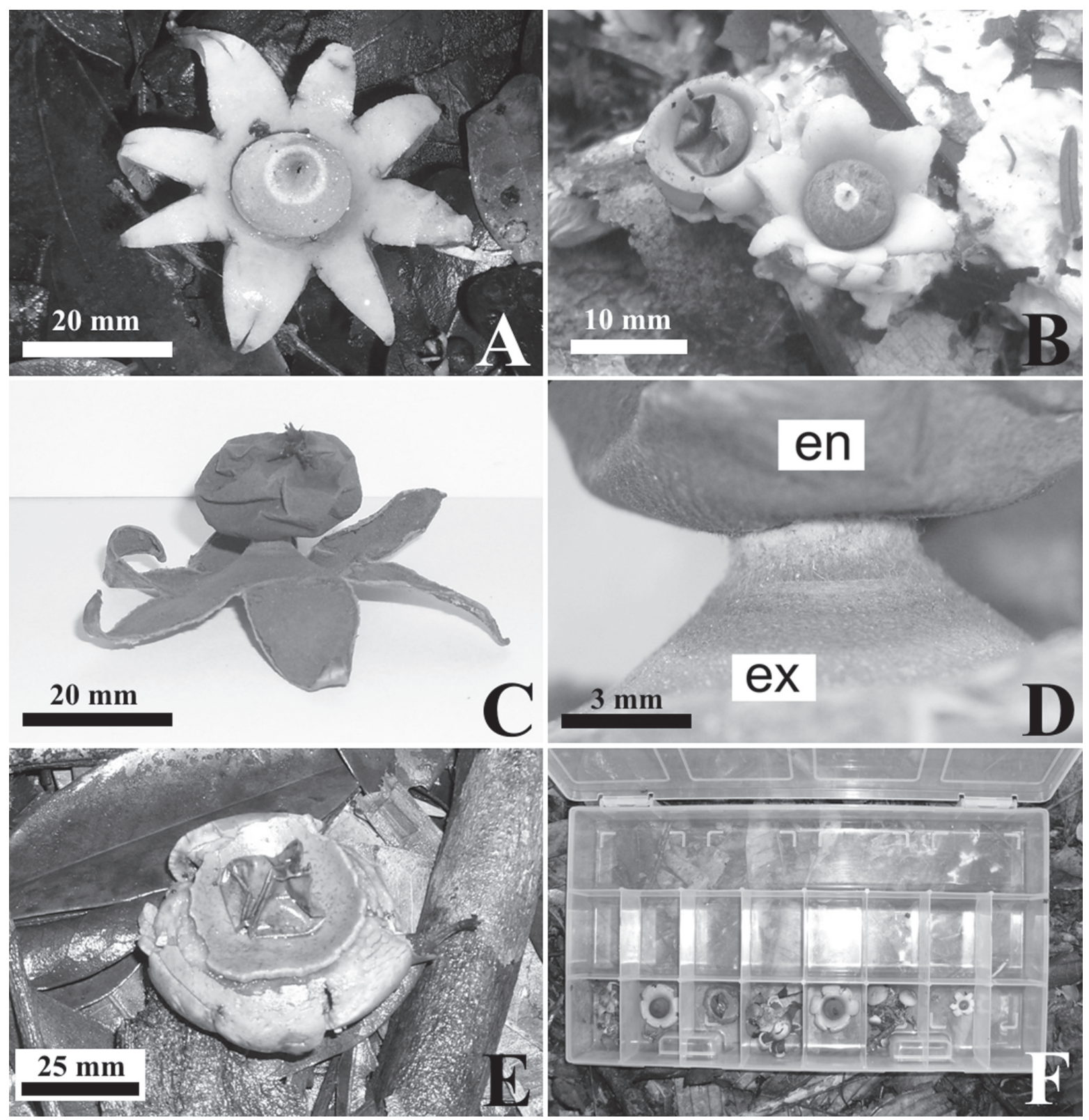

Figure 2. Basidiomata of Geastrum species. A. G. saccatum. B. G. schweinitzii. C-D. G. setiferum (paratype): C. herbarium material; D. detail of the stipe (en=endoperidium; ex=exoperidium). E. G. triplex. F. Plastic container used for collection of Geastrum specimens.

Material examined - BRAZIL. Pernambuco: Recife, DI, 13/VI/2008, Trierveiler-Pereira et al. 09 (URM 82049); V/2002, Baseia (URM 77228); Moreno, CA, 17/VI/2008, Trierveiler-Pereira \& Baltazar 20 (URM 82050); Caruaru, BC, 20/VI/2008, Trierveiler-Pereira et al. 29, 34 (URM 82052).

Taxonomic remarks - This species can be distinguished by its involute rays, prominent collar around the endoperidium, sessile endoperidium, fibrillose and delimited peristome. Other species (G. fimbriatum, G. saccatum, G. lageniforme, G. rufescens Pers.) may also show a small pseudoparenchymatous collar, but it is never as conspicuous as in G. triplex (Sunhede 1989). Basidiomata of G. triplex are usually large (up to $6.4 \mathrm{~cm}$ diam. in the examined material) and European material can reach $15 \mathrm{~cm}$ diam. (Calonge 1998).

\section{Discussion}

Macroscopic features are very important for the identification of Geastrum specimens, since the species are nearly microscopically identical when observed using light microscopy. The importance of macroscopic data to delimit species can be noticed in identification keys (Ponce de León 1968, Calonge 1998, Sunhede 1989, Bottomley 1948, Soto \& Wright 2000, Baseia et al. 2003).

Herbarium material is sometimes very difficult to determine, due to the lack of information and the poor preservation condition of important macrostructures. Therefore, some recommendations are given on collecting and improving the herborization quality of Geastrum specimens: 
1) include notes about the substrata where the specimens were collected. Geastrum species usually grow on very specific habitat;

2) when collecting, pay attention to the surroundings and look for immature basidiomata. They are often subglobose to pyriform, and may be totally or partially hypogeous (sometimes hard to find);

3) basidiomata of some species lose their external layer at maturity, as in G.hirsutum and G. albonigrum Calonge \& Mata. In this case, external layer characteristics are decisive in identifying these two species. When collecting, pay attention around mature basidioma and try to see if the external layer has peeled off;

4) when drying the material, pay special attention to the peristome. It will be impossible to analyze the peristome, and thus identify the species, if it becomes covered by folds from the endoperidium. Materials must be dried at low temperatures $\left(25-30^{\circ} \mathrm{C}\right)$ for $24-48$ hours.

\section{Acknowledgements}

The first author would like to thank CNPQ (Conselho Nacional de Desenvolvimento Científico e Tecnológico, Brazil) for providing the Master's scholarship, PROPESQ (Pró-Reitoria para Assuntos de Pesquisa e Pós-Graduação, UFPE) for financial support, and CSIC (Real Jardín Botânico de Madrid) and MA-Fungi Herbarium for all the support during her stay in Madrid. The authors also acknowledge Dra. Leonor Costa Maia, curator of URM Herbarium, for authorizing the loan of exsiccatae.

\section{References}

Baseia, I.G. \& Calonge, F.D. 2006. Geastrum hirsutum: a new earthstar fungus with a hairy exoperidium. Mycotaxon 95: 301-304.

Baseia, I.G.; Calonge, F.D. \& Maia, L.C. 2006. Notes on the Phallales in Neotropics. Boletín de la Sociedad Micológica de Madrid 30: 87-93.

Baseia, I.G.; Cavalcanti, M.A. \& Milanez, A.I. 2003. Additions to our knowledge of the genus Geastrum (Phallales: Geastraceae) in Brazil. Mycotaxon 85: 409-416.

Baseia, I.G. \& Milanez, A.I. 2002. Geastrum setiferum (Gasteromycetes): a new species with a setose endoperidium. Mycotaxon 84: 135-140.

Bottomley, A.M. 1948. Gasteromycetes of South Africa. Bothalia 4: 473-810.

Calonge, F.D. 1998. Flora Micologica Iberica vol. 3. Gasteromycetes I. Lycoperdales, Nidulariales, Phallales, Sclerodermatales, Tulostomatales. Madrid/Berlin/Stuttgart, Real Jardín Botánico de Madrid/J. Cramer.

Cortez, V.G.; Baseia I.G. \& Silveira, R.M.B. 2008a. Gasteromicetos (Basidiomycota) no Parque Estadual de Itapuã, Viamão, Rio Grande do Sul, Brasil. Revista Brasileira de Biociências, Porto Alegre 6(3): 291-299.

Cortez, V.G.; Sulzbacher, M.A.; Baseia, I.G. \& Silveira, R.M.B. 2008b. Two little known gasteroid fungi from Santa Catarina State, southern Brazil. Mycotaxon 106: 297-302.

Domínguez de Toledo, L. 1996. Geastrum lilloi sp. nov. from Argentina. Mycologia 88: 858-862.
Douanla-Meli, C.; Langer, E. \& Calonge, F.D. 2005. Geastrum pleosporus sp. nov., a new species of Geastraceae identified by morphological and molecular phylogenetic data. Mycological Progress 4(3): 239-250.

Drechsler-Santos, E.R., Wartchow, F., Baseia, I.G., Gibertoni, T.B. \& Cavalcanti, M.A.Q. 2008. Revision of the Herbarium URM I. Agaricomycetes from the semi-arid region of Brazil. Mycotaxon 104: 9-18.

Fazolino, E.P., Calonge, F.D. \& Baseia, I.G. 2008. Geastrum entomophilum, a new earthstar with an unusual spore dispersal strategy. Mycotaxon 104: 449-453.

Gomes-Silva, A.C. \& Gibertoni, T.B. 2009. Revisão do herbário URM III. Novas ocorrências de Aphyllophorales (Basidiomycetes) para Amazônia brasileira. Revista Brasileira de Botânica 32: 585-594.

Holmgren, P.K. \& Holmgren, N.H. 1998. Index Herbariorum: A global directory of public herbaria and associated staff. http://sweetgum. nybg.org/ih (accessed in: 21/05/2009).

Hosaka, K.; Bates, S.T.; Beever, R.E.; Castellano, M.A.; Colgan Iii, W.; Dominguez, L.S.; Nouhra, E.R.; Geml, J.; Giachini, A.J.; Kenney, S.R.; Simpson, N.B.; Spatafora, J.W. \& Trappe, J.M. 2006. Molecular phylogenetics of the gomphoid-phalloid fungi with an establishment of the new subclass Phallomycetidae and two new orders. Mycologia 98(6): 949-959.

Kasuya, T.; Yamamoto, Y.; Sakamoto, H.; Takehashi, S.; Hoshino, T. \& Kobayashi, T. 2009. Floristic study of Geastrum in Japan: three new records for Japanese mycobiota and reexamination of the authentic specimen of Geastrum minus reported by Sanshi Imai. Mycoscience 50: $84-93$.

Kornerup, A. \& Wanscher, J.H. 1978. Methuen Handbook of Colour. 3rd ed. London. Eyre Methuen.

Leite, A.G. \& Baseia, I.G. 2007. Novos Registros de Geastraceae Corda para o Nordeste Brasileiro. Sitientibus, Série Ciências Biológicas 7: 178-183.

Leite, A.G.; Calonge, F.D. \& Baseia, I.G. 2007a. Additional studies on Geastrum from Northeastern Brazil. Mycotaxon 101: 103-111.

Leite, A.G.; Silva, B.D.B.; Araújo, R.S.; Baseia, I.G. 2007b. Espécies raras de Phallales (Agaricomycetidae, Basidiomycetes) no Nordeste do Brasil. Acta Botanica Brasilica 21: 119-124.

Maia, L.C. 2003. Coleções de fungos nos herbários brasileiros: estudo preliminar. In: Peixoto, A.L. (Org). Coleções biológicas de apoio ao inventário, uso sustentável e conservação da biodiversidade. Rio de Janeiro, Instituto de Pesquisas Jardim Botânico do Rio de Janeiro.

Miller, O.K. Jr. \& Miller, H.H. 1988. Gasteromycetes: Morphology and Developmental Features. Eureka. Mad River.

Pereira, J.; Bezerra J.L. \& Maia, L.C. 2008. Revision of taxa of the URM Herbarium 2. Hypoxylon species described by A.C. Batista. Mycotaxon 104: 405-408.

Ponce de León, P. 1968. A revision of the Geastraceae. Fieldiana, Botany 31: 303-349.

Rick, J. 1961. Basidiomycetes Eubasidii no Rio Grande do Sul. Brasilia. Iheringia 9: 451-480.

Soto, M. \& Wright, J.E. 2000. Taxonomía del género Geastrum (Basidiomycetes, Lycoperdales) en la provincia de Buenos Aires, Argentina. Boletín de la Sociedad Argentina de Botánica 34: 185-201.

Sunhede, S. 1989. Geastraceae (Basidiomycotina). Morphology, ecology and systematics with special emphasis on the North European species. Synopsis Fungorum 1: 1-534.

Trierveiler-Pereira, L. \& Baseia, I.G. 2009a. A checklist of the Brazilian gasteroid fungi (Basidiomycota). Mycotaxon 108: 441-444.

Trierveiler-Pereira, L. \& Baseia, I.G. 2009b. Revision of the Herbarium URM IV. Nidulariaceae (Basidiomycota). Nova Hedwigia 89(3-4): 361-369.

Trierveiler-Pereira, L. \& Baseia, I.G. 2010. Additional data on Geastrum entomophilum (Geastraceae, Basidiomycota). Boletín de la Sociedad Micológica de Madrid 34: 135-139.

Trierveiler-Pereira, L.; Bezerra, K.M.T.; Bezerra, J.L. \& Baseia, I.G. 2009. First records of Geastraceae and Nidulariaceae (Basidiomycota, Fungi) from Bahia, Northeastern Brazil. Revista Brasileira de Biociências, Porto Alegre 7(3): 316-319. 